\title{
Study of Some Serum Biochemical Markers of Liver Fibrosis in Patients with Chronic HCV
}

\author{
Aly M El-Kady, Hossam El-Din F Abo-El-Kheir, Abeer Sh El-Hadidy* and Salwa El-Houfy
}

Department of Tropical Medicine and Clinical Pathology, Alexandria Faculty of Medicine, Alexandria University, Egypt

${ }^{*}$ Corresponding author: Abeer Sh El-Hadidy, Department of Tropical Medicine and Clinical Pathology, Alexandria Faculty of Medicine, Alexandria University, Egypt, Tel: +20 3 4862506; E-mail: dr-hossam1970@yahoo.com

Rec date: Feb 27, 2017; Acc date: Apr 12, 2017; Pub date: Apr 14, 2017

Citation: El-Kady AM, Abo-El-Kheir HEF, El-Hadidy AS, Study of Some Serum Biochemical Markers of Liver Fibrosis in Patients with Chronic HCV. J Clin Gastroenterol Hepatol 2017, 1: 2.

\section{Abstract}

Chronic hepatitis $\mathrm{C}$ is a major cause of cirrhosis, hepatocellular carcinoma and HCV related end stage liver disease, in many countries it is the first cause of liver transplantation. The infection is often asymptomatic, but chronic infection can lead to scarring of the liver and ultimately to cirrhosis, which is generally apparent after many years. Establishing accurate staging of liver disease is very important for enabling both therapeutic decisions and prognostic evaluations. A liver biopsy is considered the gold standard for assessing the stage of hepatic fibrosis, but it has many limitations. During the last decade, several noninvasive markers for assessing the stage of hepatic fibrosis have been developed. Some have been well validated and are comparable to liver biopsy. This paper focused on some noninvasive biochemical markers used to stage liver fibrosis. The aim of this study was to evaluate some serum biochemical markers for diagnosing liver fibrosis in patients with $\mathrm{CHC}$. This study was conducted on 50 patients who were divided into two groups: group 1 included 25 patients with F1 liver fibrosis; group 2 enrolled 25 patients with F2 liver fibrosis (according to metavir scoring system) and fit for the combination therapy (Pegyalated interferon + riba virin). Results: Serum HA. SHASTA index and FIBRO-Q LEVEL were significantly higher in patient with grade-2 fibrosis than grade F1 patients. From the previous study, we can conclude that these parameters could be useful noninvasive markers of liver fibrosis and cost-effective alternative to other serum markers for staging liver fibrosis and for determining the timing of HCV treatment.

Keywords: Chronic HCV; Liver Fibrosis; Cirrhosis; Hepatocellular carcinoma; Proteoglycans

\section{Introduction}

Chronic hepatitis $\mathrm{C}$ is a major cause of cirrhosis, hepatocellular carcinoma and HCV related end stage liver disease, in many countries it is the first cause of liver transplantation. The infection is often asymptomatic, but chronic infection can lead to scarring of the liver and ultimately to cirrhosis, which is generally apparent after many years. [1] Establishing accurate staging of liver disease is very important for enabling both therapeutic decisions and prognostic evaluations. A liver biopsy is considered the gold standard for assessing the stage of hepatic fibrosis, but it has many limitations. During the last decade, several noninvasive markers for assessing the stage of hepatic fibrosis have been developed. Some have been well validated and are comparable to liver biopsy. This paper will focus ON some noninvasive biochemical markers used to stage liver fibrosis.

Fibrosis is the result of the wound-healing response of the liver to repeated injury [2] (After an acute liver injury (e.g., viral hepatitis), parenchymal cells regenerate and replace the necrotic or apoptotic cells. This process is associated with an inflammatory response and a limited deposition of ECM. If the hepatic injury persists, then eventually the liver regeneration fails, and hepatocytes are substituted with abundant ECM, including fibrillar collagen [3]. As fibrotic liver diseases advance, disease progression from collagen bands to bridging fibrosis to frank cirrhosis occurs. it is associated with major alterations in both the quantity and composition of ECM. In advanced stages, the liver contains approximately 6 times more ECM than normal, including collagens (I, III, and IV), fibronectin, undulin, elastin, laminin, hyaluronan, and proteoglycans. Accumulation of ECM results from both increased synthesis and decreased degradation [4]. Hepatic Stellate Cells (HSCs) are the main ECM-producing cells in the injured liver [5]. Following chronic injury, HSCs activate or trans-differentiate into myofibroblast-like cells, acquiring contractile, pro-inflammatory, and fibrogenic properties [6]. Activated HSCs migrate and accumulate at the sites of tissue repair, secreting large amounts of ECM and regulating ECM degradation. Collagen synthesis in HSCs is regulated at the transcriptional and posttranscriptional levels [7] Apoptosis of damaged hepatocytes stimulates the fibrogenic actions of liver myofibroblasts. Inflammatory cells, either lymphocytes or polymorpho-nuclear cells, activate HSCs to secrete collagen

The assessment of fibrosis in liver diseases provides much information not only for the diagnosis and prognosis of disease, but also for the therapeutic decision and for the monitoring and evaluation of treatment. The methods for assessing liver fibrosis include: Invasive methods using liver 
biopsy, Noninvasive methods (Fibrotic markers (Direct serological markers and indirect serological markers), imaging methods (Ultrasonography (US), Computed tomography (CT, Magnetic resonance imaging (MRI))) and Fibro scan.

Despite the development of potential diagnostic tests for fibrosis assessment, needle biopsy of the liver remains the gold stanat provides much useful information [8]. Liver biopsy remains the best way to assess the severity of chronic hepatitis $\mathrm{C}$, to determine the prognosis and to evaluate the indication of therapy. The risk of developing cirrhosis depends on the stage (degree of fibrosis) and the grade (degree of inflammation and necrosis) observed on the initial liver biopsy [8].

Unfortunately, liver biopsy has some limitations and complications, including sample errors which is present in about $20 \%$ of liver biopsies, it may miss advanced fibrosis in $30 \%$ of patients [9-11], pain which is the most frequent risk of percutaneous liver biopsy, bleeding which is encountered in one per 1000 liver biopsies, infection, trauma to internal organs and death [12]. There are several methods to read a liver biopsy. The most common scoring methods are known as the Knodell, Ishak and Metavir Scores. It is important to remember that the size of the piece of liver taken at biopsy can affect the report. The knowledge of the doctor reading the biopsy is also important.

Biomarkers of fibrosis are commonly divided into direct and indirect markers. Direct markers are fragments of the liver matrix components produced by hepatic stellate cells (HSC) during the process of ECM remodeling. Indirect markers include molecules released into the blood due to liver inflammation, molecules synthesized/regulated or excreted by the liver, and markers of processes commonly disrupted due to liver function impairment. Direct and indirect markers may be used alone or more commonly - in combination with each other, to produce composite scores. The calculation of such scores can be relatively simple or can be based on complicated formulas [13]. These markers are directly involved in fibrogenesis and fibrolysis. They include markers of matrix metabolism as well as cytokines. Serum levels of direct markers reflect simultaneously both processes as well as the total mass of ECM undergoing remodeling [14]. The assessment of direct markers could be useful for staging liver disease and for assessing the effect of treatment and predicting disease progression.

They offer an attractive, cost effective alternative to liver biopsy for both patients and clinicians. In addition to being substantially less invasive, there are practically no complications, little or no sampling errors and small observer related variability. Moreover, measurements may be performed repeatedly, thus, allowing for a dynamic monitoring of fibrosis. The ideal marker for liver fibrosis should have the following characteristics: Be highly sensitive and specific to identify different stages of fibrosis, be readily available, safe, inexpensive and reproducible and be applicable to the monitoring of disease progression or regression as a part of natural history of liver disease or treatment regimens. Although no single ideal marker exists, several markers have been identified as possible useful indicators of fibrosis when used in conjunction with each other individual test $[15,16]$.

\section{Study Design}

This study was conducted on 50 patients who were divided into two groups: group 1 included 25 patients with F1 liver fibrosis; group 2 enrolled 25 patients with F2 liver fibrosis (according to metavir scoring system) and fit for the combination therapy (pegyalated interferon +ribavirin). All patients were subjected to informed consent obtained from all patients before sampling, thorough history taking including risks for acquiring infection, any treatment received before, investigations done before and any associated medical diseases and clinical evaluation. Laboratory investigations including routine investigations (Complete blood picture, fasting blood glucose level and serum urea and creatinine). Liver function tests including serum alanine transaminase (ALT), serum aspartate transaminase (AST), serum albumin, serum bilirubin (total, direct), prothrombin activity, alkaline phosphatase (ALP) and gamma glutamyl transferase (GGT). Specific investigations: Viral markers: Hepatitis $C$ Virus antibodies (HCV Abs) [17]. Hepatitis B surface antigen (HBsAg), HCV RNA quantitative polymerase chain reaction (PCR) was done [18], Ultrasound guided liver biopsy was done for patients.

\section{Medical Imaging (Abdominal Ultrasonography)}

Serum Hyaluronic acid (HA) by enzyme-linked immunosorbant assay (ELISA) using specific ELISA kit (produced by USCN Life Science Inc.) which is a competitive inhibition enzyme immunoassay technique for the in vitro quantitative Hyaluronan. $\mathrm{HA}$ is a glycosaminoglycan synthesized by HSCs and it is a component of the ECM. High levels of HA in serum may reflect increased synthesis of ECM by HSCs, and it appears to be the best individual test that reflects ECM concentration HA normal range $0-75 \mathrm{ng} / \mathrm{mL}$ [19].

The aspartate aminotransferase (AST)/ALT (AAR) ratio is one of the eldest markers of liver fibrosis that is easily available and applicable, AST/platelet ratio (APRI) was developed by Wai et al. in 2003 and is measured as APRI=AST level (/ULN) * 100/ platelet count [20] FIB-4 score: based on age, platelet count, AST, and ALT. FIB4=age ${ }^{*}$ AST/PLT ${ }^{*}$ VALT. Fibro-Q test was proposed by Hsieh et al. in 2009. It is calculated based on age, AST, prothrombin time (PT-INR), platelet count, and ALT [21]. SHASTA index is based on serum hyaluronic acid, AST, and albumin [22]. SHASTA index $=3.84+1.7$ (if $4<\mathrm{HA}<85 \mathrm{ng} / \mathrm{mL}$, otherwise 3.28 ) +1.58 (if albumin $<3.5 \mathrm{mg} / \mathrm{dl}$, otherwise 0 ) + 1.78 (if $A S T>60 \mathrm{IU} / \mathrm{L}$, otherwise 0) [23]. API index=age/PLT index. 


\section{Aim of the study}

The aim of this study was to evaluate some serum biochemical markers for diagnosing liver fibrosis in patients with $\mathrm{CHC}$.

\section{Statistical Analysis}

Statistics of the results were carried out according to chisquare (X2) for comparison between distributions of patients according to different items of the study. $\mathrm{P}<0.05$ was considered statistically significant. All analyses were done using SPSS version 16.0 for Windows statistical software (SPSS Inc., Chicago, IL, USA). Sensitivity relates to the test's ability to identify a condition correctly. Specificity relates to the test's ability to exclude a condition correctly. There are several terms that are commonly used along with the description of sensitivity, specificity and accuracy. They are true positive (TP), true negative (TN), false negative (FN), and false positive (FP). They are described in terms of TP, TN, FN and FP.

Sensitivity $=$ TP $/(T P+F N)=(\#$ true positive assessment $) /(\#$ all positive assessment).

Specificity $=$ TN $/(T N+F P)=(\#$ true negative assessment $) /$ (\#all negative assessment).

Accuracy $=(\mathrm{TN}+\mathrm{TP}) /(\mathrm{TN}+\mathrm{TP}+\mathrm{FN}+\mathrm{FP})=($ \#correct assessments)/\#all assessments).

\section{Results}

In group 1 (F1), the mean of serum hyaluronic acid level was 37.4 with a standard deviation of 11.6 while in group 2 (F2) it was 22.7 with a standard deviation of 16.1. The mean of serum hyaluronic acid was significant higher in group 2 than in group 1 with $P$-value $=0.00$ and $R$-value $=0.11$. In group 1 , the mean of APRI was 0.34 with a standard deviation of 0.11 while in group 2 it was 0.35 with a standard deviation of 16.1. The mean of APRI was not significantly higher in group 2 than in group 1 ( $P$ value $=0.93, \mathrm{R}$-Value $=0.18$ ). Moreover, in group 1 , the mean of SHASTA was 6.89 with a standard deviation of 0.89 while in group 2 it was 7.14 with a standard deviation of 1.04. Then mean of SHATA index in group 2 was significantly higher than group 1 ( $P$-value $=0.0017, R$-value $=0.2$ ). As regard AST-ALT ratio, the mean was 0.81 in group 1 with a standard deviation of 0.03 while in group 2 it was 0.83 with a standard deviation of 0.04. There was no significant correlation as regard AST/ALT ratio among both groups ( $P$-value $=0.83, R$-value $=0.11$ ). However, in group 2, the mean of FIB4 was 0.85 with a standard deviation of 0.38 while in group 1 it was 0.68 with a standard deviation of 0.15 . There was no significant correlation between both groups ( $P$-value=0.74, R-value=0.3). On the other hand, in group 1, the mean of FIBRO-Q was 1.03 with a standard deviation of 0.27 while in group 2 it was 1.37 with a standard deviation of 0.35 . There was a positive but not strong significant correlation among both groups ( $P$ value $=0.03$, R-value $=0.34)$. Furthermore, in group 1 , the mean of API was 0.14 with a standard deviation of 0.03 while in group 2 it was 0.16 with a standard deviation of 0.04 . There was no significant correlation among both groups as regard API (P-value $=0.75, \mathrm{R}$-value $=0.18$ ) as shown in Tables $\mathbf{1}$ and $\mathbf{2}$.

Table 1 Statistical analysis for the fibrosis markers.

\begin{tabular}{|c|c|c|c|c|c|c|c|c|c|c|c|c|c|c|}
\hline & \multicolumn{2}{|l|}{ HA } & \multicolumn{2}{|c|}{ APRI } & \multicolumn{2}{|c|}{ SHASTA } & \multicolumn{2}{|c|}{ AST-ALT } & \multicolumn{2}{|l|}{ FIB4 } & \multicolumn{2}{|c|}{ FIBRO- Q } & \multicolumn{2}{|l|}{ API } \\
\hline & $\mathrm{F} 1$ & $\mathrm{~F} 2$ & $\mathrm{~F} 1$ & $\mathrm{~F} 2$ & $\mathrm{~F} 1$ & $\mathrm{~F} 2$ & $\mathrm{~F} 1$ & $\mathrm{~F} 2$ & $\mathrm{~F} 1$ & $\mathrm{~F} 2$ & $\mathrm{~F} 1$ & $\mathrm{~F} 2$ & $\mathrm{~F} 1$ & $\mathrm{~F} 2$ \\
\hline Mean & 22.7 & 37.4 & 0.34 & 0.35 & 6.82 & 7.14 & 0.81 & 0.83 & 0.68 & 0.85 & 1.03 & 1.37 & 0.14 & 0.16 \\
\hline Std. Dev & 11.6 & 16.1 & 0.11 & 0.19 & 0.89 & 1.04 & 0.03 & 0.04 & 0.15 & 0.38 & 0.27 & 0.35 & 0.03 & 0.04 \\
\hline P-value & \multicolumn{2}{|l|}{0} & \multicolumn{2}{|l|}{0.93} & \multicolumn{2}{|c|}{0.0017} & \multicolumn{2}{|l|}{0.83} & \multicolumn{2}{|l|}{0.74} & \multicolumn{2}{|l|}{0.03} & \multicolumn{2}{|l|}{0.75} \\
\hline R-value & \multicolumn{2}{|l|}{0.11} & \multicolumn{2}{|l|}{0.18} & \multicolumn{2}{|l|}{0.2} & \multicolumn{2}{|l|}{0.11} & \multicolumn{2}{|l|}{0.3} & \multicolumn{2}{|l|}{0.34} & \multicolumn{2}{|l|}{0.18} \\
\hline
\end{tabular}

Table 2 The statistical results (cut-off value, sensitivity, specificity, accuracy, PPV and NPV) among different studied markers.

\begin{tabular}{|c|c|c|c|c|c|c|c|}
\hline & HA & APRI & SHASTA & AST-ALT ratio & FIB4 & FIBRO-Q & API \\
\hline Cut-off value & 48 & 0.55 & 7 & 0.8 & 0.9 & 1.6 & 0.17 \\
\hline Sensitivity & $60 \%$ & $33 \%$ & $100 \%$ & $100 \%$ & $16 \%$ & $21 \%$ & $100 \%$ \\
\hline Specificity & $48 \%$ & $47 \%$ & $76 \%$ & $30 \%$ & $50 \%$ & $48 \%$ & $10 \%$ \\
\hline Accuracy & $48 \%$ & $46 \%$ & $50 \%$ & $48 \%$ & $40 \%$ & $47 \%$ & $42 \%$ \\
\hline PPV & $50 \%$ & $20 \%$ & $67 \%$ & $80.30 \%$ & $10 \%$ & $70 \%$ & $50.90 \%$ \\
\hline NPV & $96 \%$ & $93 \%$ & $93 \%$ & $100 \%$ & $88 \%$ & $95 \%$ & $80 \%$ \\
\hline
\end{tabular}




\section{Discussion}

The liver is the largest organ in the body and performs many functions that are critical for survival. These functions are impaired in patients with chronic liver disease, critically so in patients with ESLD. Regardless of the underlying etiology of chronic liver disease, hepatic fibrosis is a wound-healing process in response to an acute or chronic liver injury to parenchymal cells [2]. Chronic inflammation of the liver can lead to cirrhosis, a stage of organ dysfunction and damage in which scar tissue replaces normal functioning tissue and causes a dramatic, and potentially fatal, decline in liver function. Traditionally, liver biopsy has been considered the gold standard to diagnose and monitor the progression of fibrosis in patients with chronic viral hepatitis and other liver diseases. However, liver biopsy is an invasive procedure associated with a risk of complications such as sampling errors especially with small sized biopsies, intra-observer and interobserver variation, pain and bleeding [12]. For this reason, liver biopsy has poor tolerance, particularly if it needs to be repeated over time in an individual patient.

Despite the aforementioned limitations, there has been a clear resistance to accept noninvasive diagnosis of liver fibrosis as a viable and preferable alternative to liver biopsy. The reasons for this are various. First, there is a paucity of welldesigned studies assessing noninvasive methods, and sufficient external validation for some of the proposed methods is lacking. Second, the number of proposed methods to assess disease severity remains in a state of constant growth and there is practically no time to validate or test them all. Third, liver biopsy itself is not an ideal gold standard. Finally, there is still significant opposition to changing what has long stood as dogma. The aforementioned reasons may explain why the introduction of noninvasive methods in clinical practice is making such slow headway in the field of hepatology.

NIMs are helpful in assessing the stage of fibrosis in patients with no clear indication for a liver biopsy, such as patients with chronic hepatitis $B(C H B)$ and persistently normal serum alanine aminotransferase (ALT), patients with chronic hepatitis $\mathrm{C}(\mathrm{CHC})$ or $\mathrm{CHB}$ and who require follow-up assessment of the stage of fibrosis during or after treatment, and autoimmune hepatitis $(\mathrm{AlH})$ patients who require assessment after prolonged immunosuppressive therapy [23]. The rapid development of new medications for the treatment of some liver diseases, such as $\mathrm{CHB}, \mathrm{CHC}$, and nonalcoholic fatty liver disease (NAFLD), increases the requirement for more frequent evaluation of liver fibrosis to assess treatment response. Liver biopsies are not ideal for frequent evaluations. The ideal NIM for assessing hepatic fibrosis must be simple, readily available, reliable, inexpensive, safe, and well validated in different forms of chronic liver disease. It must also be useful in assessing the progression of liver disease [24].

In our study, we investigate a direct marker of liver fibrosis which is serum hyaluronic acid and six indirect markers which are APRI, SHASTA, and AST-ALT ratio, FIB4, FIBROQ and API. We found that serum level of hyaluronic acid was significantly higher in group 2 than in group 1 with a cut-off level $\geq 48$ $\mathrm{ng} / \mathrm{mL}, \mathrm{P}$-value $=0.0001$ and $\mathrm{R}=0.51$ with a NPV $\geq 96 \%$ and specifity $48 \%$. A similar study was done by Sakugawa et al. and Murawaki et al. [25] who had defined a cutoff level of $\geq 50$ $\mathrm{ng} / \mathrm{mL}$ for detection of severe fibrosis; in another study Montazeri et al. used a cut off level of $126.4 \mathrm{ng} / \mathrm{mL}$ [26]. HA has been studied in CHC, NAFLD, alcoholic liver disease (ALD), and $\mathrm{CHB}$, but it has been more extensively studied in the former two diseases. HA has been of great value in detecting advanced fibrosis [27]. HA shows a negative predictive value of 98-100\% for cirrhosis and is of great value in excluding cirrhosis [27]. In treated $\mathrm{CHC}$ patients, the response to treatment was reported to be associated with a reduction in serum HA levels [28].

As regard, the APRI index in our study, it shows a significant higher level in group-2 than group-1 with a cut-off level $\geq 0.5$, a NPV $\geq 93 \%$ and specificity $47 \%$. In the original study, the APRI of more than 1.5 showed an area under the receiver operating curve (AUC in the ROC) of 0.8 , and showed an area under the receiver operating curve (AUC in the that APRI at a cutoff 0.42 or less correctly detected mild fibrosis with a NPV of 95\% [29]. In contrast, some studies showed that the APRI is only of moderate accuracy in assessing fibrosis in $\mathrm{CHC}$ [28].

However, SHASTA index was significantly higher in group 2 than in group 1 with a cut-off value $=7$, a negative predictive value of $\geq 97 \%$ and specificity $76 \%$ for diagnosing liver fibrosis. In contrast, in a study of $95 \mathrm{HIV} / \mathrm{HCV}$ co-infected patients, an index of 0.3 showed a sensitivity of $>88 \%$ and a negative predictive value of $>94 \%$, and a level of 0.8 showed a specificity of $100 \%$ and a positive predictive value of $100 \%$ for detection of severe fibrosis of F3 or more [29]. Using this index only, $42 \%$ of patients were correctly classified, whereas the remaining $58 \%$ showed values between 0.3 and 0.8 [29].

On the other hand, FibroQ test proposed by Hsieh et al. in 2009 [21] using a cutoff value of 1.6 the AUC for the detection of significant fibrosis was 0.783 , and the negative predictive value was $100 \%$ for the exclusion of cirrhosis. These values were both higher than those obtained when using the APRI and AAR in the same cohort [28]. More recently, a similar study showed that Fibro-Q was superior to FIB-4, AAR, APRI, and Lok's model in predicting significant fibrosis in patients with chronic hepatitis C [30]. This was matched with FIBRO-Q in our study as it was significantly higher in group 2 than group 1 correlated with a cutoff value of 1.6 , the NPV was $\geq 95 \%$ and specificity of $48 \%$.

Moreover AST-ALT ratio was not significantly different among both groups with a cutoff value of 0.8 , a negative predictive value of $\geq 100 \%$ and specificity $30 \%$. This was not matched with Sterling et al. in 2008 that showed NPVs of $96 \%$ and $81.3 \%$ and showed an enhanced performance [31].

Lastly, as regard, the FIB-4 score which was first developed by Sterling et al. to assess fibrosis in HIV/HCV confected patients at a cutoff value of $3.25 ; 87 \%$ of patients were 
correctly classified, with an AUC of 0.765 for significant fibrosis [31]. FIB-4 score in our study was not statistically significant different among both groups with a cutoff value of 0.9 and a NPV $\geq 88 \%$ and specificity of $50 \%$.

\section{Conclusion}

From the previous study, we can conclude that Serum HA, SHASTA index and FIBROQ level could be a useful non-invasive marker of liver fibrosis. They could be considered as a costeffective alternative to other serum markers for staging fibrosis and for determining the timing of HCV treatment. Moreover, Serum HA is specific, so, in the diagnosis of hepatic fibrosis, after exclusion of other diseases that increased its level. On the other hand, when diagnosis was based on a combination of those three markers, the diagnostic sensitivity for fibrosis was much increased and the combination of these approaches as first-line assessment of liver fibrosis may allow liver biopsy to be avoided in the majority of patients with chronic hepatitis $\mathrm{C}$.

\section{References}

1. Ryan KJ, Ray CG (2004) Sherris medical microbiology. (4th edn). New York: McGraw Hill, NY, USA.

2. Friedman SL (2003) Liver fibrosis-from bench to bedside. J Hepatol 38: S38-S53.

3. Pinzani M (1999) Liver fibrosis. Springer Semin Immunopathol 21: 475-490.

4. Arthur MJ (2000) Fibrogenesis II metalloproteinases and their inhibitors in liver fibrosis. Am J Physiol Gastrointest Liver Physiol 279: G245-G249.

5. Gabele E, Brenner DA, Rippe RA (2003) Liver fibrosis: Signals leading to the amplification of the fibrogenic hepatic stellate cell. Front Biosci 8: D69-D77.

6. Marra F (1999) Hepatic stellate cells and the regulation of liver inflammation. J Hepatol 31: 1120-1130.

7. Lindquist JN, Marzluff WF, Stefanovic B (2000) Fibrogenesis III posttranscriptional regulation of type I collagen. Am J Physiol Gastrointest Liver Physiol 279: G471-G476.

8. Silva MA, Hegab B, Hyde C, Guo B, Buckels JA, et al. (2008) Needle track seeding following biopsy of liver lesions in the diagnosis of hepatocellular cancer: a systematic review and meta-analysis. Gut 57: 1592-1596.

9. Bedossa P, Dargere D, Paradis V (2003) Sampling variability of liver fibrosis in chronic hepatitis C. Hepatology 38: 1449-1457.

10. Guido M, Rugge M (2004) Liver biopsy sampling in chronic viral hepatitis. Semin Liver Dis 24: 89-97.

11. Al KB, Shiffman M (2007) Percutaneous liver biopsy in clinical practice. Liver Int 279: 1166-1173.

12. Piccinino F, Sagnelli E, Pasquale G, Giusti G (1986) Complications following percutaneous liver biopsy. A multicentre retrospective study on 68, 276 biopsies. J Hepatol 22: 165-173.

13. Grigorescu M (2006) Non-invasive biochemical markers of liver fibrosis. J Gastrointestin Liver Dis 15: 149-159.
14. Pinzani $\mathrm{M}$, Rombouts $\mathrm{K}$, Colagrande $\mathrm{S}$ (2005) Fibrosis in chronic liver disease: Diagnostic and management. J Hepatol 42: S12S36.

15. Zhou K, Lu LG (2009) Assessment of fibrosis in chronic liver diseases. J Dig Dis 10: 714

16. Afdhal NH, Nunes D (2004) Evaluation of liver fibrosis: A concise review. Am J Gastroenterol 99: 1160-1174.

17. Colin C, Lanoir D, Touzet S, Meyaud-Kraemer L, Bailly F, et al. (2001) Sensitivity and specificity of third-generation hepatitis C virus antibody detection assays: an analysis of the literature. $J$ Viral Hepat 8: 87-95.

18. Scott JD, Gretch DR (2007) Molecular diagnostics of hepatitis C virus infection: A systematic review. JAMA 297: 724-732.

19. McHutchison JG, Blatt LM, De Medina M, Craig JR, Conrad A, et al. (2000) Measurement of serum hyaluronic acid in patients with chronic hepatitis $C$ and its relationship to liver histology. Consensus Interferon Study Group. J Gastroenterol Hepatol 15: 945-951.

20. Wai CT, Greenson JK, Fontana RJ, Kalbfleisch JD, Marrero JA, et al. (2003) A simple noninvasive index can predict both significant fibrosis and cirrhosis in patients with chronic hepatitis C 1. Hepatology 38: 518-526.

21. Hsieh YY, Tung SY, Lee IL, Lee K, Shen CH, et al. (2009) FibroQ: An easy and useful non-invasive test for predicting liver fibrosis in patients with chronic viral hepatitis. Chang Gung Medical Journal 32: 614-622.

22. Cales P, Oberti F, Michalak S, Hubert-Fouchard I, Rousselet MC, et al. (2005) A novel panel of blood markers to assess the degree of liver fibrosis. Hepatology 42: 1373-1381.

23. Leroy V, Hilleret MN, Sturm N, Trocme C, Renversez JC, et al. (2007) Prospective comparison of six non-invasive scores for the diagnosis of liver fibrosis in chronic hepatitis C. J Hepatol 46: 775-782.

24. Nobili V, Parkes J, Bottazzo G, Marcellini M, Cross R, et al. (2009) Performance of ELF serum markers in predicting fibrosis stage in pediatric non-alcoholic fatty liver disease. Gastroenterology 136: 160-167.

25. Sakugawa H, Nakayoshi T, Kobashigawa K, Yamashiro T, Maeshiro T, et al. (2005) Clinical usefulness of biochemical markers of liver fibrosis in patients with non-alcoholic fatty liver disease. World Journal of Gastroenterology 11- 255-259.

26. Montazeri G, Estakhri A, Mohamadnejad M, Nouri N, Montazeri $F$, et al. (2005) Serum hyaluronate as a non-invasive marker of hepatitic fibrosis and inflammation in $\mathrm{HBeAg}$ negative chronic hepatitis B. BMC Gastroenterol 5: 32.

27. McHutchison JG, Blatt LM, Medina MD, Craig JR, Conrad A, et al. (2000) Measurement of serum hyaluronic acid in patients with chronic hepatitis $C$ and its relationship to liver histology. Journal of Gastroenterology and Hepatology 15: 945-951.

28. Rosenberg WM, Voelker $M$, Thiel $R$, Becka $M$, Burt $A$, et al. (2004) Serum markers detect the presence of liver fibrosis: A cohort study. Gastroenterology 127: 1704-1713.

29. Kelleher TB, Mehta SH, Bhaskar R, Sulkowski M, Astemborski J, et al. (2005) Prediction of hepatic fibrosis in HIV/HCV co-infected patients using serum fibrosis markers: The SHASTA index. Journal of Hepatology 43: 78-84. 
30. Hsieh YY, Tung SY, Lee K, Shyong Wu C, Wei KL et al. (2012) Routine blood tests to predict liver fibrosis in chronic hepatitis $\mathrm{C}$. World Journal of Gastroenterology 18: 746-753.

31. Sterling RK, Lissen E, Clumeck N, Sola R, Correa MC, et al. (2006) Development of a simple noninvasive index to predict significant fibrosis in patients with HIV/HCV co-infection. Hepatology 43: 1317-1325. 